\title{
Research Progress on Regulatory T Cell Differentiation and Regulation
}

\author{
Jing Feng ${ }^{1}$, Zhaoxin $\mathrm{Mu}^{2}$, Zhenjiang $\mathrm{Hou}^{2, *}$ \\ ${ }^{1}$ Cangzhou Central Hospital, Cangzhou, China \\ ${ }^{2}$ Institute of Thyroid Diseases Affiliated to Cangzhou Medical College, Cangzhou Thyroid Disease Engineering Technology Research Center, \\ Cangzhou, China
}

Email address:

houzhenjiang@sina.com (Zhenjiang Hou)

${ }^{*}$ Corresponding author

\section{To cite this article:}

Jing Feng, Zhaoxin Mu, Zhenjiang Hou. Research Progress on Regulatory T Cell Differentiation and Regulation. American Journal of Clinical and Experimental Medicine. Vol. 8, No. 3, 2020, pp. 25-34. doi: 10.11648/j.ajcem.20200803.11

Received: April 10, 2020; Accepted: May 29, 2020; Published: June 8, 2020

\begin{abstract}
Regulatory T cells (Treg) are a subset of T cells with immunosuppressive functions. According to the source and mechanism of Treg cells, they are divided into natural regulatory $\mathrm{T}$ cells (nTreg) and induced regulatory $\mathrm{T}$ cells (iTreg). According to the different properties of secreted cytokines, they are divided into two types: pro-inflammatory and anti-inflammatory cells. Pro-inflammatory Treg cells that secrete IFN-ã are closely related to the development of autoimmune diseases, while anti-inflammatory Treg cells that secrete IL-10 can reduce the development of slow inflammation. In addition to secreting a variety of inhibitory cytokines such as IL-10 and TGF-â, Treg cells also exert immunosuppressive effects through direct contact among cells to jointly maintain the body's immune tolerance and suppress immune responses. By suppressing the autoimmune response of effector $\mathrm{T}$ cells and promoting immune tolerance, it has a very important role in maintaining the homeostasis of the body. The differentiation and regulation of Treg cells and their relationship with autoimmune diseases have been hotspots in the field of immunology in recent years. This article reviews the source of Treg, differentiation regulation, classification, markers, functions and mechanisms of action so as to deepen the understanding of Treg cell differentiation and immune regulation mechanisms, and further broaden the research horizon and thinking.
\end{abstract}

Keywords: Regulatory $\mathrm{T}$ Cells, Cluster of Differentiation $4^{+} \mathrm{T}$ Cells, Interleukin-10, Transforming Growth Factor- $\beta$, Forkhead Box p3, Signal Transduction and Activator of Transcription

\section{Introduction}

T cells are derived from bone marrow lymphoid stem cells, which differentiate, develop and mature in the thymus, mainly including Cluster of differentiation (CD) $4^{+}$and $\mathrm{CD}^{+} \mathrm{T}$ cells. The former accounts for about $65 \%$ of $\mathrm{T}$ cells, play an important role in immunity and assist in humoral immune response [1]. $\mathrm{T}$ lymphocytes are one of the most important immune cells, and their subgroups have many names. According to the different functions of the immune response, it is currently recognized that they can be divided into helper $\mathrm{T}$ cells that help cellular immunity and humoral immune function (helper $\mathrm{T}$ cell, $\mathrm{Th}$ ), regulatory $\mathrm{T}$ cells (Treg) that regulate the immune response, and cytotoxic $\mathrm{T}$ lymphocytes (CTL) with the function of killing target cells.
After being induced by $\mathrm{T}$ cell receptor (TCR) and induced by cytokines, $\mathrm{CD}^{+} \mathrm{T}$ cells are mainly divided into 4 cell subpopulations: Th1, Th2, Th17, and Treg. Th1 and Th2 cells are the first discovered $\mathrm{CD} 4{ }^{+} \mathrm{T}$ cell subsets and Th1/Th2 immunization model is a classic immunology theory. Treg and Th17 originated from the same type of initial $\mathrm{CD}^{+} \mathrm{T}$ cells. They have a close and complex relationship, which restricts each other in differentiation and antagonizes in function, and can be transformed into each other under certain conditions. Both of them constitute a relatively independent cell network system except Th1/Th2. Tregs are a type of $\mathrm{T}$ cell subset with immunosuppressive function, which plays a vital role in maintaining the immune tolerance of the body. It can regulate CD4+, CD8+ T cells, B cells, and natural killer cells (NK) in the body, natural killer $\mathrm{T}$ cells (NKT) and different antigen presenting cells (Antigen 
presenting cells, APC) and other immune responses of various cells. Tregs have a variety of different regulatory mechanisms, and can adopt different immune regulation strategies [2] according to different microenvironments, which play an important role in the immune system. By inhibiting the activation and proliferation of other immune cells, it exerts its effects of inducing immune suppression and immune incompetence. Forkhead box p3 (Forkhead box p3, Foxp3) is a specific transcription factor of Treg, and its continuous expression can promote the differentiation of Treg cells and improve the immunosuppression [3]. Studies have confirmed that Treg cells and their cytokines and transcription factors are closely related to the maintenance of the body's immune homeostasis and the occurrence and development of autoimmune diseases (AID). The reduction in the number of Tregs cells or functional defects will lead to the occurrence of AID [4], and became a hot spot for AID occurrence, outcome and treatment.

\section{Discovery and Differentiation regulation of Treg Cells}

\subsection{Discovery of Treg Cells}

As early as the 1970s, people realized that there was a class of immunosuppressive $T$ cell subsets in the body. In the 1980 s, a class of $\mathrm{T}$ cell subsets with unique functions were discovered, which played an important role in preventing autoimmune reactions. It was not until 1995 that Sakaguchi et al. [5] first isolated $\mathrm{CD} 4^{+} \mathrm{CD} 25^{+} \mathrm{T}$ cell subsets in mouse spleen and peripheral blood, namely, due to their continuous function of expressing CD25 molecule, $\mathrm{CD} 4^{+} \mathrm{T}$ cell subsets that had characteristic of expressing interleukin 2 receptor (IL-2R). Treg cells usually referred to a subset of $\mathrm{CD}^{+} \mathrm{T}$ lymphocytes that highly expressed CD25 and Foxp3, can inhibit the activation of autoreactive $\mathrm{T}$ cells, and participate in the regulation of immune responses and immune tolerance. Knocking out $\mathrm{CD} 4^{+} \mathrm{CD} 25^{+} \mathrm{T}$ cells in mice can quickly induce autoimmunity and multiple organ damage in mice, and reinfusion of $\mathrm{CD} 4{ }^{+} \mathrm{CD} 25^{+} \mathrm{T}$ cells can reverse or prevent the occurrence of autoimmune reactions [6]. Therefore, it is believed that $\mathrm{CD} 4^{+} \mathrm{CD} 25^{+} \mathrm{T}$ cells have dual functions of immunoregulation and immunosuppression, so concept of Tregs was first proposed that Treg cells had the function of inhibiting the activation of other T cells. Later, similar results in the human body were confirmed. In 2001, Stephens et al. found natural $\mathrm{CD} 4{ }^{+} \mathrm{CD} 25^{+}$Treg cells in human thymus and peripheral blood, the content of which accounted for $5 \%$ to $15 \%$ of $\mathrm{T}$ cells. In recent years, studies have found that Treg cells can perform immunosuppressive regulation by secreting interleukin 10 (interleukin, IL-10) and transforming growth factor- $\beta$ (Transforming growth factor- $\beta$, TGF- $\beta$ ). Foxp 3 is the best specific marker for Treg cells. CD $4^{+} \mathrm{CD} 25^{+}$Foxp3 Treg cells are currently widely used specific surface molecular markers for Tregs. They are considered to be the most representative cell subgroup of Treg cells [7]. Foxp3 plays a key role in the development and regulation of Treg cells. Reduced Treg number or loss of function affect the body's immune status, causing autoimmune tolerance disorders [8] and leading to the occurrence of AID.

\subsection{Differentiation Regulation of Treg Cells}

Both Treg and Th17 cells are derived from the same type of initial $\mathrm{CD}^{+} \mathrm{T}$ cells. After differentiation into intermediate cells, the direction of their differentiation depends mainly on the type of cytokines in the environment, and they transform into each other under certain conditions. TGF- $\beta$ mediates the differentiation of $\mathrm{T}$ cells into Treg. After adding IL-6, the differentiation is stopped, and the differentiation to Th17 direction. Treg cell differentiation is inhibited to a certain extent, showing that IL-6 plays a decisive role in the direction of T cell differentiation. Large amounts of IL-6 and IL-21 can also transform differentiated Treg cells into Th17 cells. Another study showed that TGF- $\beta$ played an important role in the differentiation of $\mathrm{T}$ cells into Th17 or Treg cells [9]. Low levels of TGF- $\beta$ and IL- 6 together promote the differentiation of Th17 cells. High concentrations of TGF- $\beta$ acting alone can induce high Foxp3 expression, promote Treg cell differentiation, and cause $\mathrm{CD} 4^{+} \mathrm{CD} 25^{+}$Treg cells to inhibit Th17. IL-17 and IL-6 can effectively inhibit the function of Th1 and transform Treg cells to Th17 cells. In the presence of IL-10, Th17 cells can be transformed into Treg cells. The differentiation of Treg cells was regulated by many factors. Under neutral conditions in vitro, TGF- $\beta$ would tend to balance towards the development of functional Foxp $3^{+}$Tregs. In the presence of inflammatory cytokines such as IL-1 $\beta$, IL-2, IL-15, functional Tregs were transformed into non-functional Tregs that produced IL-17. IL-2 was a key factor in regulating the homeostasis of Treg cells, and immature Treg cells can be used to increase the expression of Foxp3 and CD25 and promote differentiation into mature Treg cells. IL- 2 and TGF- $\beta$ induced the differentiation of naive T cells into Treg cells. IL-2 induced STAT5, enhanced Foxp 3 expression, and promoted Treg differentiation. TGF- $\beta$ was mainly involved in the differentiation process of Treg through STAT5 induction, and retinoic acid activated the differentiation process by promoting TGF- $\beta$ and inducing Foxp3 [10]. IL-35 was a new anti-inflammatory factor secreted by Treg cells after IL-10 and TGF- $\beta$. It had stronger immunological functions than IL-10 and TGF- $\beta$ and played a negative immunoregulatory role in the differentiation of Treg cells [10]. IL-35 could inhibit the proliferation of T cells, expand Treg cells, and induce iTr35 with stronger inhibitory activity to exert its immunomodulatory effect [11]. iTr35 could secrete IL-35 specifically, inhibit the proliferation of T cells, and was a key factor in inducing infectious tolerance [12], effectively preventing the occurrence and development of inflammation. The differentiation state of $\mathrm{CD} 4^{+} \mathrm{T}$ cells was mainly determined by environmental factors and their transcription factors [13]. Under the stimulation of different cytokines, $\mathrm{CD} 4^{+} \mathrm{T}$ cells can be differentiated into different effector $\mathrm{T}$ cells. The latter and Treg restricted each other in the process of function and differentiation, and jointly maintained the immune homeostasis of the body. The 
imbalance of immune will lead to the occurrence of immune-related diseases $[13,14]$.

\section{Classification and Markers of Treg Cells}

\subsection{Classification of Treg Cells}

At present, the classification of the Tregs subgroup has not been unified. According to the origin, differentiation and mechanism of Treg, they are divided into two categories: natural regulatory $\mathrm{T}$ cells (nTregs) and induced regulatory $\mathrm{T}$ cells (iTregs). The vast majority of nTreg cells are derived from the thymus, which is mediated by endogenous superantigens and APCs derived from thymus and bone marrow, and induced differentiation, development and maturation by TCR, cytokines, cell surface receptors and costimulatory molecules, and then migrate to peripheral tissues. It is an intrinsic Treg cell produced by normal procedures in the thymus and also known as intrinsic or thymus-type Tregs (tTregs). In peripheral blood, nTreg mainly expresses CD4, CD25, Foxp3, PD-1, CCR4 and low levels of CD127, among which Foxp3 is considered to be the most specific marker for human and mouse Treg cells. iTreg, also known as adaptive regulatory $\mathrm{T}$ cells or peripheral Tregs (pTregs), is derived from the differentiation of peripheral primary $\mathrm{CD} 4^{+} \mathrm{T}$ cells through antigens or cytokines such as IL-10, TGF- $\beta$, and $1 \mathrm{~L}-2$. iTreg is divided into two categories: one is the type I regulatory $\mathrm{T}$ cells characterized by IL-10 and dendritic cells (DCs) that do not express Foxp3, but highly express IL-10, namely $\operatorname{Tr} 1\left(\mathrm{CD}^{+} \mathrm{CD} 25\right.$ low Foxp3 $\left.{ }^{-}\right)$, mainly through the secretion of IL-10 to suppress the initial and memory Thl and Th2 cell responses [15]. The other type is a subset of regulatory $\mathrm{T}$ cells $\left(\mathrm{CD} 4^{+} \mathrm{Treg}\right)$ cells that specifically express Foxp 3 induced by TGF- $\beta 1$, namely Th3 $\left(\mathrm{CD} 4^{+} \mathrm{CD} 25^{-}\right.$Foxp3) cells, mainly through self-secreted TGF$\beta 1$, suppresses Th1 cell-mediated immune response and inflammatory response [16]. Two subgroups of iTreg derived from naive resting $\mathrm{T}$ cells after DCs stimulation, depending on the type and activation state of DCs. In fact, nTreg and iTreg cells do not have very strict boundaries, and there is a certain overlap. Generally speaking, $\mathrm{CD} 4{ }^{+} \mathrm{CD} 25^{+}$Treg cells mainly refer to nTreg. In addition, Tregs cells also include CD8 ${ }^{+}$Treg, CD4 $4^{-} \mathrm{CD} 25^{-}$Treg, $\mathrm{CD}^{+}{ }^{+} \mathrm{LAP}^{+}$Treg and NKT [17]. $\mathrm{CD} 8^{+}$Treg in humans is mainly derived from $\mathrm{CD} 8^{+} \mathrm{CD} 28^{+} \mathrm{T}$ lymphocytes, and NKT cells express NK cell receptors NK1.1 or NK161 in addition to T cell receptor TCR $\alpha \beta$ [18].

\subsection{Markers of Treg Cells}

Many scholars believe that Foxp3 plays a key role in the development and function of Treg, and its expression determines the maturity and functional regulation of nTregs. It has been recognized by immunologists as a specific biomolecule marker to activate nTregs. Foxp3 is a traditionally recognized specific marker for $\mathrm{CD} 4^{+} \mathrm{CD} 25$ Treg cells. With the deepening of research, it has found that Foxp3 is not only specifically expressed in nTregs, but also expressed in human activated Teff. Therefore, the rationality of Foxp3 as a specific biological marker for activated human nTregs has been questioned, and doing research on multiple markers of Treg cell subpopulations has been carried out. The immunophenotype of Tregs also includes CD127low, CD45RO, CD45RA, etc [19]. According to the positive expression of CD25 molecule on the surface of $\mathrm{CD} 4^{+} \mathrm{T}$ cells, it can be divided into $\mathrm{CD} 4^{+} \mathrm{CD} 25^{+}$regulatory $\mathrm{T}$ cells (Treg) and $\mathrm{CD} 4^{+} \mathrm{CD} 25^{-}$effector $\mathrm{T}$ cells (Teff), the former can suppress the immune response, and the latter can promote immune response. According to the phenotype of cells, the Treg subgroup mainly includes $\mathrm{CD} 4^{+} \mathrm{CD} 25^{+}$, $\mathrm{CD}^{+} \mathrm{CD} 25^{+} \mathrm{CD}_{22} \mathrm{~L}^{+}, \quad \mathrm{CD} 4^{+} \mathrm{CD} 25^{+} \mathrm{CD} 103^{+}$, $\mathrm{CD} 4^{+} \mathrm{CD} 25^{+} \mathrm{CD} 122^{+}, \quad \mathrm{CD} 4^{+} \mathrm{CD} 152^{+}, \quad \mathrm{CD} 4^{+} \mathrm{CD} 103^{+}$, $\mathrm{CD} 4^{+} \mathrm{CD} 25^{+}$Foxp 3 and $\mathrm{CD} 4^{+} \mathrm{CD} 45 \mathrm{RB}$ low Treg cells, among which $\mathrm{CD} 4{ }^{+} \mathrm{CD} 25^{+}$Treg cells are currently the most studied, account for about $5 \% \sim 10 \%$ of peripheral blood $\mathrm{CD}^{+} \mathrm{T}$ cells [20], which can maintain autoimmune balance and prevent the occurrence of AID. According to the expression of CD25 molecule, it can be used as the main basis for distinguishing Th cells and Treg cells, but the expression of CD25 on the surface of Th cells after infection and activation can be weak to moderate. Therefore, CD25 cannot be used as a specific marker for $\mathrm{CD}^{+} \mathrm{CD} 25^{+}$Treg cells $[21,9]$. According to the combination of Poxp3 and CD45RA, Treg cells are divided into resting type (Poxp3 dim CD45RA ${ }^{+}$) and activated type (Poxp3 high CD45RA-). nTreg cells may have a full set of $\mathrm{T}$ cell receptor lineages that recognize their own antigens, also known as antigen non-specific Treg cells [22]. IL-2, CD28 and TCR/CD3 complex are necessary factors to maintain the growth and proliferation of nTreg cells. According to the expression and level of CD45RA and Foxp3, human peripheral blood Tregs can be divided into activated type (CD4 ${ }^{+}$CD45RA Foxp3 high activated, aTregs), secreted (IFN- $\gamma$, IL-2) type (CD4 ${ }^{+} \mathrm{CD} 25^{+} \mathrm{CD} 45 \mathrm{RA}^{-}$Foxp3 low) and resting type $\left(\mathrm{CD}^{+} \mathrm{CD} 45 \mathrm{RA}^{+}\right.$Foxp3 low resting, rTregs) [11], these three types of cells have different expansion and inhibition capabilities, the first two can exert effective inhibition in vitro, while the latter cannot, indicating that Foxp3 alone cannot determine the true inhibitory Tregs. Using flow cytometry to detect Foxp3 in cells can identify human peripheral blood $\mathrm{CD} 4^{+} \mathrm{CD} 25^{+}$Treg cells. However, as Foxp3 is a nuclear protein existing in cells, it needs to be detected after membrane rupture staining. In addition, due to individual differences and cumbersome operation, the separated $\mathrm{CD} 4{ }^{+} \mathrm{CD} 25^{+}$Foxp $3^{+}$Treg cells are not suitable for the detection of cell function [12], and the activated CD $4^{+} \mathrm{CD} 25^{-}$Tcells can also express Foxp [13], which indicates that not all Foxp3 positive cells are $\mathrm{CD} 4{ }^{+} \mathrm{CD} 25^{+}$Foxp3 Treg cells. CD127 (the alpha chain of IL-7 receptor) is another marker on the surface of human Treg cells. Foxp3 may be a "switch" for Treg cells to start the immunosuppressive function, which can inhibit the promoter of CD127, and over 90\% express Foxp3. CD127+subpopulation CD127 showed low expression, namely, CD4+CD25+Foxp3+low expression CD127 Treg 
cells indicating that $\mathrm{CD} 127$ and Foxp3 expression are negatively correlated, $\mathrm{CD} 4^{+} \mathrm{CD} 25^{+} \mathrm{CD} 127^{-} /$low Treg and $\mathrm{CD} 4^{+} \mathrm{CD} 25^{+}$Foxp 3 Treg cells may have the same effect in the body, that is, highly inhibit the function of Teff. Since C127 is a specific molecular marker on the surface of Treg cells, it is expressed at high levels in naive and mature T cells, but at low levels in Treg cells. Combined detection of CD25 and CD127 can sort Treg cells for cell function testing [14]. Compared with Foxp3, the detection of $\mathrm{CD} 4^{+} \mathrm{CD} 25^{+} \mathrm{CD} 127-/$ lowTreg cells is relatively simple, and it is more advantageous to detect Treg cells by flow cytometry. The combined detection of CD4, CD25 and CD127 can not only determine the Treg cells of $\mathrm{CD} 4^{+} \mathrm{CD} 25^{+} \mathrm{CD} 127^{-} /$low, but also improve the purity of Treg cells, making it a type of Treg cell subgroup with higher specificity and easier to quantify. However, because Teff also expresses CD4 and $\mathrm{CD} 25$, and even the subpopulation of $\mathrm{T}$ cells with high expression of $\mathrm{CD} 25$ contains $50 \%$ of the newly activated $\mathrm{T}$ cells, it is difficult for Treg cells to effectively screen Teff. Therefore, the two markers CD4 and CD25 are not enough to distinguish Treg from conventional $\mathrm{T}$ cells, especially from Teff. In recent years, based on the discovery of cell surface marker latency-Associated peptide (LAP), it has become a new subset of Treg cells with immunosuppressive function, which is named $\mathrm{LAP}^{+} \mathrm{CD} 4^{+} \mathrm{T}$ Cells [23] is a new marker of non-Foxp $3^{+}$suppressor cells in human peripheral blood. It can be mediated by IL-10 and TGF- $\beta$ in vitro to inhibit the proliferation of other T cells. Scurr et al [24] believed that the $\mathrm{LAP}^{+} \mathrm{CD} 4^{+}$Treg subgroup had stronger immunosuppressive activity than the classical $\mathrm{CD} 4^{+} \mathrm{CD} 25^{+} \mathrm{Foxp}^{+} \mathrm{T}$ cells. As a propeptide, LAP was noncovalently bound to the amino terminal of TGF- $\beta$ to form a latent TGF- $\beta$ complex, which can be expressed in the cell membranes of various immune cells such as Tregs, DCs and platelets. There were also reports of Foxp3 expression in CD4 ${ }^{+} \mathrm{LAP}$ Treg cells in the peripheral blood of healthy people, and there was no report or a small amount of expression in each subgroup of $\mathrm{CD}^{+}{ }^{+} \mathrm{LAP}^{+}$Treg [25]. Recent studies have found that glycoprotein A repetitions predominant (GARP) transmembrane proteins are considered to be specific surface markers for activating Tregs [26], and are only selectively expressed in human activated nTreg, enhancing Treg Cell function, but not expressed in activated Th cells, is currently considered to be a specific marker for human activation of nTreg cells. GARP (LRRC32) is mainly expressed on the surface of activated Foxp $3^{+}$Tregs. CD $25^{+} \mathrm{GARP}^{+}$Tregs have stronger immunosuppressive capacity than CD $25^{+}$GARP ${ }^{-}$Tregs. More than $90 \%$ of $\mathrm{CD}^{2} 5^{+} \mathrm{GARP}^{+} \mathrm{T}$ cells simultaneously express Foxp3 [18], and less than 3\% of Teffs cells also express GARP. The expression of CD154 on the surface of Tregs is low, and the inhibitory ability of Tregs combined with GARP is significantly higher than that of $\mathrm{CD} 4^{+} \mathrm{CD} 25$ hiTregs and $\mathrm{CD} 4^{+} \mathrm{CD} 25^{+} \mathrm{CD} 127$ lowTregs [26]. Most $\mathrm{CD}^{+} \mathrm{CD} 25^{+} \mathrm{GARP}^{+} \mathrm{T}$ cells express Foxp3, and only $1 \%-2 \%$ express low levels of IL-17 and IFN- $\gamma$, while $\mathrm{CD} 4^{+} \mathrm{CD} 25^{+} \mathrm{GARP}^{-} \mathrm{T}$ cells express high levels of IL-17. It is suggested that GARP can not only identify Tregs with higher inhibitory function, but also isolate Th17 cells from $\mathrm{CD} 4^{+} \mathrm{CD} 25^{+} \mathrm{T}$ cells, which improves the purity of Tregs. Therefore, GARP may be a surface marker of activated Tregs, which can be combined with other surface molecules to sort high-purity Tregs cells. $\mathrm{CD} 4^{+} \mathrm{CD} 25^{+}$Treg cells can also express a variety of non-specific markers, such as cytotoxic $\mathrm{T}$ lymphocyte-associated antigen-4 (CTLA-4), glucocorticoid-induced tumor necrosis factor (glucocorticoid-Induced tumor necrosis factor receptor, GITR), programmed death ligand-1 (Programmed death-ligand1, PD-L1), CD45RO, etc. are expressed in both Treg cells and Teff, so they cannot be used as specific molecules for Treg cells Mark [18]. A study [27] found that GARP can directly bind to the LAP of TGF- $\beta$ on the surface of nTreg and upregulate the levels of LAP and GARP in active Treg cells. However, the use of Foxp3-siRNA to downregulate Foxp3 did not affect the expression of GARP and LAP. Therefore, Foxp3 in Treg cells was not necessary for GARP and LAP expression. At present, there are also methods for isolating and purifying the Treg cell subpopulation, removing CD127 cells with high levels of expression in the $\mathrm{CD}^{+} \mathrm{CD} 25^{+}$cell subpopulation, can concentrate Foxp3 high expression and high expansion potential Tregs subtypes, and jointly use anti-CD49d and CD127 antibody is used to extract high-purity, unlabeled Tregs with inhibitory effect, and its clinical application value needs to be further verified. Therefore, so far, Treg cells still lack more specific markers. On the basis of previous research, according to the principles of evidence-based laboratory medicine, CD4, CD25, CD127, Foxp3, LAP and GARP are optimized and combined, and according to the ROC curve, molecular markers with high sensitivity and specificity of Treg cells are determined imperative.

\section{Function and Mechanism of Action on Treg Cells}

\subsection{Function of Treg Cells}

Tregs are a subset of $\mathrm{T}$ cells with immunosuppressive effects, mainly by inhibiting the activation of other T cells to exert immune regulation, inducing immune tolerance, and playing an important role in maintaining immune homeostasis and suppressing autoimmune reactions [28]. Treg cells can induce the production of cytokines in chronic inflammation, inhibit the effect of Teff, mediate immune tolerance and prevent tissue damage, and provide the necessary guarantee for the body's overactive immune response. $\mathrm{CD}^{+} \mathrm{CD} 25^{+}$Treg is a class of immunosuppressive $\mathrm{T}$ cell subsets. On the one hand, it secretes cytokines such as IL-10 and TGF- $\beta$ to maintain the stability of the immune system; on the other hand, it can inhibit the reaction of IL-17 and protect the body free from damage caused by inflammatory reactions [29]. NTregs directly differentiated from thymocytes function through intercellular contact, while iTreg cells, which are transformed from peripheral mature $\mathrm{CD} 4^{+} \mathrm{CD} 25^{+} \mathrm{T}$ cells through specific antigen stimulation and 
cytokine induction, mainly play an immunosuppressive role by secreting inhibitory cytokines such as il-10 and TGFinhibition [30]. Treg cells secrete IL-10, TGF- $\beta$ and IL-35 and other cytokines to suppress the body's autoimmune response [31], mainly manifested as two functions of immune anergy (anergic) and immune suppression (immunity suppressive) [32]: (1) Immune impotence means that Treg cells do not respond to high-concentration IL-2 alone and the combined application of anti-CD3 and anti-CD28 monoclonal antibodies, namely, various stimulations cannot make Treg cells proliferate and differentiate, nor secrete IL- 2. Even under the joint action of TCR and IL-2, the proliferation and differentiation ability of Treg cells is also weak [33]; (2) Immunosuppression means that Treg cells are activated by TCR-mediated and high-concentration exogenous IL-2 in combination, further inhibiting the differentiation and proliferation of $\mathrm{CD} 4+$ and CD8+T cells, hindering the APC-mediated effect, resulting in the death of target cells. Its inhibitory effect is non-antigen specific. nTreg mediates inhibition and regulation through cell non-contact mechanisms, maintains the body's autoimmune homeostasis and immune tolerance, and controls inflammatory responses. The functions of iTreg and nTreg cells are similar. They mainly play a negative immunoregulatory role and suppress the immune function of the body. pTregs cells have more specificity than allogenic reactive $\mathrm{T}$ cells. However, the epigenetics of nTreg and iTreg are different. The former has the effect of inhibiting the activation and function of APCs such as DCs, Teff, B cells and monocytes [19]. Foxp3 expression is more stable and demethylation is more extensive. Data shows that iTreg is more effective than nTreg in inhibiting the activation of autoimmune cells, which may be related to the former's greater stability in the inflammatory environment [20]. Compared with CD39-iTreg cells, CD39 ${ }^{+}$iTreg shows stronger proliferation and inhibitory capacity, and its inflammatory cytokine expression is lower [34].

\subsection{Mechanism of Action on Treg Cells}

Due to surface Treg cells express a variety of molecules, cells and secrete numerous inhibitory factor and so on, through the combination of surface molecules, inhibitory cytokine secretion and release of toxic substances, inhibition of antigen presenting cells, dendritic cells induced resistance, outside secrete body immunity, etc. and immune cells and their factors influence each other, its mechanism of immunosuppression may be the interaction of multiple approaches, constitute the immune regulation network of complex and huge system, complete the body's immunity. However, the immunosuppressive mechanism of Treg cells is not fully understood, and there may be several ways: (i) Through the mechanism of inhibitory cytokines, Treg cells secrete sex cytokines such as IL-10, IL-35 or TGF- $\beta$ to inhibit the effect of Teffs and to prevent excessive immune response in the body [35]. The inhibitory effect of the above cytokines on Treg cells is very important, and this pathway is considered to be the main inhibitory mechanism. In vivo experiments show that the inhibitory effect of IL-10 and TGF- $\beta$ on Tregs is essential. IL-10 mainly inhibits the function of APC, and only depends on IL-10 in the innate immune system. As a soluble cytokine, TGF- $\beta$ can be expressed on the surface of activated Tregs, and can function by membrane contact. IL-35 is mainly expressed by Foxp $3+$ nTregs, which is necessary for Tregs to exert their maximum inhibitory activity. Foxp3 expression is significantly up-regulated in Tregs in the inhibitory role. IL-35 is ectopically expressed in sufficient amounts to make the original $\mathrm{T}$ cells rich in regulation activity, after recombination can inhibit the proliferation of $\mathrm{T}$ cells. After Foxp3+Treg is activated by TCR-mediated signal stimulation, it can inhibit the activation and proliferation of Foxp3-T cells by secreting inhibitory cytokines, IL-2 and other competitive depletion. Foxp3+Treg can also contact with target cells, secrete IL-10, TGF $\beta$ and IL-35, and exert the function of suppressing immune cells [32]. Under the effect of TGF- $\beta$ and IL-6, naive $T$ cells can preferentially differentiate into Teffs, which reduces Tregs. TGF- $\beta$ can also inhibit IL- 1 and IL-2, suppress immune cell activation and Teffs function. (ii) Through intercellular contact mechanism: Treg cells are in contact with Teffs, which promotes the combination of CTLA-4 on the surface of Treg cells and CD80 and CD86 on the surface of Teffs, blocking the signal pathway, which can inhibit the generation of the second signal, make $\mathrm{T}$ cells immune disabled, and induce Immune tolerance exerts immunosuppressive effects [36]. The CTLA-4, GITR, PD-L1 and HLA-DR expressed by Treg cells induce the costimulatory factor (ICOS), and cross-link with APC to suppress the activity of immune cells, thereby exerting an immunosuppressive effect [37]. The high expression of GITR in Treg cells not only inhibits its immune activity, but also reduces the expression of FoxP3 in vivo, which negatively regulates the immunosuppression of Treg cells [38]. Overexpression of CTLA-4 on the surface of Treg cells, interacts with CD80 and CD86, promotes DC expression of indoleamine 2, 3-dioxygenase (IDO), inhibits activation of $\mathrm{T}$ cells and induces apoptosis [39]. It can also stimulate the expression of IDO in DC and induce the production of resistant IDO-DC cells. The increase in the number can inhibit the antigen presentation of DC cells [40]. The interaction between IDO and Treg cells may be the key to immune tolerance One of the mediating factors. (iii) Through cell lysis mechanism most studies believe that Treg cells promote the lysis of cells and mediate Teffs cell apoptosis by releasing the synergistic effect of granzyme $\mathrm{B}(\mathrm{GrB})$ and perforin (PFP). PFP causes target cells to form transmembrane channels. GrB is transported into target cells through the channels formed by PFP, and apoptosis is induced by osmotic contrast. GrB can also induce apoptosis of target cells through various ways. PFP can enhance GrB's Pro-apoptotic effect. The cell lysis mechanism induced by Tregs is mediated by granzyme $\mathrm{B}$, which is perforin-dependent, and it may be that Tregs itself have cytolytic activity. GrB expressed by activated Treg cells can kill various target cells such as mature or immature DC, 
$\mathrm{CD}^{+} / \mathrm{CD}^{+} \mathrm{T}$ cells through PFP-dependent cytotoxicity [41]. Studies have also shown that activated Tregs induce ligand death pathway through TNF- $\alpha$ and induce Teffs apoptosis. It has also been suggested that galectin mediates cell lysis in a GrB and PFP-dependent manner. GrB and PFP may also induce the release of cytokines in the particle-mediated lysis pathway. $\mathrm{CD} 4^{+} \mathrm{CD} 25^{+}$Treg cells express granzyme A under the synergy of $\mathrm{CD} 3$ and $\mathrm{CD} 46$ antibodies, killing activated $\mathrm{CD} 4^{+} \mathrm{T}$ cells and $\mathrm{CD} 8^{+} \mathrm{T}$ cells [42]. (iv) Through dendritic cell-mediated mechanism Tregs affect the activation and immunosuppression of Teffs by regulating DC maturation and function. Tregs regulate DC maturation and function by inhibiting surface molecule lymphocyte activation gene (LAG) 3 and CTLA-4, directly affect the immunosuppressive effect of Teffs, and can also inhibit the maturation of APCs or induce tolerance Sexual DCs play an immunosuppressive effect [43]. MHC II expressed by mature DC binds to LAG-3, which can induce an inhibitory signaling pathway mediated by the immunoreceptor tyrosine activation motif (ITAM). SHP1 inhibits the maturation of DC and downregulates its immune activation ability. iIL-35 can induce Treg cells to express the co-inhibitory molecule CTLA-4 and APCs surface marker molecules CD80 and CD86 with high affinity binding to initiate the inhibitory signal. After $\mathrm{T}$ cells receive antigen stimulation, they proliferate and differentiate to form Teffs [44]. Recent studies have found that the low expression of immunomodulatory molecules such as CD40, CD80, CD86, CD45RA, CD45RA ${ }^{+}$CD45RB, MHC-II, and OX62 on the surface of DC cells can inhibit the immune response ability of DC cells [45]. Adenosine can also regulate the maturation of DC and induce the differentiation of Foxp3 and Tregs. Leukocyte function-associated antigen-1 (LFA-1) can accelerate theaccumulation of Tregs and immature DCs. By relying on LFA-1 and CTLA-4, it can down-regulate the expression of CD80 and CD86 on DCs. DC loses its ability to activate naive $\mathrm{T}$ cells, leading to immunosuppression and immune tolerance. Fibrinogenfi-like protein 2 (FGL2) secreted by Tregs may affect the function of DC. The application of its antibody can completely block the inhibitory effect of Tregs. FGL2 binds to the low-affinity Fc RIIB receptor, inhibits DC maturation, and induces B cell apoptosis. The number of DCs in FGL2-/-mice increased. After stimulation with LPS, the expressions of MHC-II and CD80 were up-regulated, and the number of Tregs increased, but its inhibitory effect decreased. Tregs can express neuropilin 1 (neuropilin 1, NRP1), which may promote the interaction between Tregs and immature DC. Blocking Nrp1 weakens the effect of Tregs and DC. Foxp $3^{+}$Treg can negatively regulate APC by inhibiting antigen presentation, reducing costimulatory signals, etc., resulting in high expression of LAG-3/CD233 on the cell membrane, which combines with APC's MHC II to inhibit DC activation [46] and block DC maturation. Treg can also inhibit the maturation of APC or induce tolerance DC to play an immunosuppressive effect [43]. (v) By inhibiting the metabolic mechanism and interfering with the metabolism of
$\mathrm{T}$ cells, such as the glycoprotein receptor on the surface of $\mathrm{T}$ cells reacts with galectin, which can reduce the IL-2 and interferon gamma secreted by $\mathrm{T}$ cells, leading to metabolic disorders. The high expression of the IL-2 receptor CD25 on the surface of Tregs cells causes Teffs to be competitively bound to IL-2 and is consumed. By depleting the IL-2 that Teffs depends on to survive, it inhibits its proliferation and apoptosis. The highly expressed CD39 and CD73 molecules on the surface of Treg cells cleave extracellular ATP, catalyze the production of adenosine, further activate the adenosine A2A receptor, and promote the increase and adaptability of cyclic adenosine monophosphate (cAMP) in Teffs. The proliferation of Tregs interferes with its metabolic and biological functions and inhibits Teffs. In addition, galectin depletion of IDO by Treg cells reduces the local tissue tryptophan and inhibits the proliferation of T cells [47]. IDO plays an important role in the process of Treg's appreciation, differentiation and apoptosis. Tregs combine with CTLA-4 and CD80/CD86 with high affinity to increase the content of IDO in DC cells, induce the catabolism of tryptophan into pro-apoptotic metabolism, enhance the immunosuppressive capacity of cells, and suppress Teffs. The IFN- $\gamma$-IDO axis is essential for the production and function of Treg. Treg can promote the expression of IDO and enhance its activity. Through the feedback pathway between Treg and IDO, the interaction affects the immune tolerance environment. IDO also produces metabolites by breaking down tryptophan, binding to the cytoplasmic transcription factor aromatic hydrocarbon receptor (AhR), causing naive $\mathrm{CD} 4+\mathrm{T}$ cells to differentiate into Treg and Th17 cells. AhR also induces human DC to produce IDO in the feedback loop, further inhibiting $\mathrm{T}$ cell proliferation. (vi) The research on the mechanism of exosome production shows that Treg cells fuse with the target cells to release the content through the generated exosomes, or internalize the target cells to take up the content, or bind to specific proteins on the surface of the target cells [48], Regulating the function of Teffs by paracrine or endocrine methods to maintain autoimmune tolerance, and play the role of immune regulation [49]. Treg cell-derived exosomes contain various components from the cell itself, such as cytokines, membrane protein surface molecules, and RNA, and exert an immunosuppressive effect through high-efficiency interactions with target cells. Exosomes released after activation of $\mathrm{CD} 8^{+} \mathrm{CD} 25^{+}$Treg cells can be expressed in molecules such as CD25 and GITR. Agarwal et al. [50] found that exosomes derived from Treg cells can express CD25 and CTLA-4 at high levels, and induce immune tolerance by inhibiting the proliferation and activity of Teffs cells to exert an immunosuppressive effect. The CD73 molecule expressed by Treg exosomes activates cAMP in Teffs through the adenosine/adenosine receptor pathway, promoted immune tolerance, and inhibits the effect of Teffs. Treg cells were interacted with CTL by releasing exosomes to suppress DC antigenpresenting cells and had the effect of inhibiting $\mathrm{T}$ cell proliferation. Chatila et al. believed that the secretion of exosomes by Treg cells can induce immune 
tolerance [51]. The exosomes secreted by Treg cells contain unique miRNAs and iNOS molecules, which can induce apoptosis and promote the transformation of $\mathrm{T}$ cells to Treg [52]. Treg cell-derived exosome miRNAs suppress gene production and self-reactive $\mathrm{T}$ cells through gene silencing, or by containing inhibitory cytokines and surface molecules bound to target cells, suppress immune responses, participate in the maintenance of autoimmune processes, regulation and immune tolerance [53]. Aiello et al. [60] blocked the NF- $\mathrm{BB}$ signaling pathway by adenovirus vector transfer dominant suppressor gene nuclear factor kinase inhibitor (IKK2), and the immature DC obtained induced Treg cells in vitro, and the exosomes contained miRNA, interfered with $\mathrm{T}$ cell cycle protein expression, and inhibited $\mathrm{T}$ cell proliferation. Exosomes contained NO synthase (iNOS), which induced the production of large amounts of $\mathrm{NO}$, leading to apoptosis of Teffs cells. The same amount of $\mathrm{CD} 4{ }^{+} \mathrm{CD} 25^{+}$Foxp $3^{+}$Treg cells secreted more exosomes than other immune cells such as Teffs and Th17, and Treg cells were more sensitive to DC exosomes than $\mathrm{DC}^{+}$and $\mathrm{CD}^{+}$Subtypes of $\mathrm{T}$ cells [54]. Studies have found that exosomes secreted by Treg cells have a similar effect to Treg cells, but their inhibitory ability is weaker than Treg cells themselves [55], inferring that exosomes are only a way for Treg cells to exert an immunosuppressive effect. It is not a decisive role in complementing its high-efficiency immune regulation [56]. Therefore, the regulatory mechanism of exosomes secreted by Treg cells needs further study. (vii) Notch signaling pathway is highly conserved. It activates receptors through transmembrane ligands and plays an important role in multiple steps and links. It participates in $\mathrm{T}$ cell activation and its mediated immune response [57]. The differentiation of cells is crucial, which can promote the $\mathrm{Th} 2$ response and regulate the differentiation of $\mathrm{T}$ cells towards Th1. Notch signaling pathway not only plays a key role in the differentiation of Th cells, but also plays an immunosuppressive role by regulating the differentiation and expression of Treg cells [58]. Notch1 binds to its ligand, induces Treg differentiation, regulates the number of Treg cells, and mediates immunosuppression. Inhibiting Notch signal can significantly promote Treg to secrete IL-10 and other anti-inflammatory cytokines, and inhibit the expression of IL-1 $\beta$ pro-inflammatory cytokines. Notch signaling pathway can also regulate the immunosuppressive function of Tregs, enhance the signal response of Th1 cells, down-regulate the stability of peripheral Foxp3+Treg [59], participate in the regulation of FoxP3+Treg in PBMC, block Notch signaling pathway, FoxP3 expression is significantly down-regulated This indicates that Notch signal has a greater influence on FoxP3 expression [60]. (viii) Foxp3 determines the differentiation and maintenance of Treg cells through the transcription factor mechanism [61]. The interruption of its expression can hinder the differentiation of Treg. Foxp3 and $\mathrm{T}$ cells act to inhibit the activity of Teffs and NKT. (ix) Through gap connection mechanism Tregs cells can connect through gaps to transfer the inhibitory second messenger cAMP to Teffs cells and DC, inhibiting Teffs to play a role. (x) Through Treg cell activation mechanism Treg cells are activated by TCR and can directly inhibit Teffs without being mediated by APC. That is, Treg cells directly "talk" with Teffs. In short, the mechanism of action of Treg cells is quite complex, which may be dominated by one pathway, or may be the result of the interaction of multiple pathways.

\section{Application and Prospect of Treg Cells}

$\mathrm{T}$ cells are derived from bone marrow lymphoid stem cells, which differentiate, develop and mature in the thymus, mainly including Cluster of differentiation (CD) $4^{+}$and $\mathrm{CD}^{+} 2 \mathrm{~T}$ cells. The former accounts for about $65 \%$ of $\mathrm{T}$ cells, play an important role in immunity and assist in humoral immune response. $\mathrm{T}$ lymphocytes are one of the most important immune cells, and and their subgroups have many names. According to the different functions of the immune response, it is currently recognized that they can be divided into helper $\mathrm{T}$ cells that help cellular immunity and humoral immune function (helper T cell, Th), regulatory $\mathrm{T}$ cells (Treg) that regulate the immune response, and cytotoxic T lymphocytes (CTL) with the function of killing target cells. After being induced by $\mathrm{T}$ cell receptor (TCR) and induced by cytokines, $\mathrm{CD}^{+} \mathrm{T}$ cells are mainly divided into 4 cell subpopulations: Th1, Th2, Th17, and Treg. Th1 and $\mathrm{Th} 2$ cells are the first discovered $\mathrm{CD} 4^{+} \mathrm{T}$ cell subsets and Th1/Th2 immunization model is a classic immunology theory. Treg and Th17 originate from the same type of initial $\mathrm{CD}^{+} \mathrm{T}$ cells. They have a close and complex relationship, which restricts each other in differentiation and antagonizes in function, and can be transformed into each other under certain conditions. Both of them constitute a relatively independent cell network system except Th1/Th2. Tregs are a type of $\mathrm{T}$ cell subset with immunosuppressive function, which plays a vital role in maintaining the immune tolerance of the body. It can regulate $\mathrm{CD}^{+}, \mathrm{CD}^{+} \mathrm{T}$ cells, $\mathrm{B}$ cells, and (natural killer cells in the body. NK), natural killer T cells (NKT) and different antigen presenting cells (Antigen presenting cells, APC) and other immune responses of various cells. Tregs have a variety of different regulatory mechanisms, and can adopt different immune regulation strategies according to different microenvironments, which play an important role in the immune system. By inhibiting the activation and proliferation of other immune cells, it exerts its effects of inducing immune suppression and immune incompetence. Foxp3 is a specific transcription factor of Treg, and its continuous expression can promote the differentiation of Treg cells and improve the immunosuppression. Studies have confirmed that Treg cells and their cytokines and transcription factors are closely related to the maintenance of the body's immune homeostasis and the occurrence and development of autoimmune diseases (AID). The reduction in the number of Tregs cells or functional defects will lead to the occurrence of AID, and became a hot spot for AID occurrence, outcome and treatment. 


\section{Acknowledgements}

Cangzhou Key R \& D Plan and Guidance Project (Project No: 1833020011).

Natural Science Foundation of Cangzhou Medical College (No. 18Z015).

\section{References}

[1] Ma XH, Xiao L. Research progress in members of lymphocyte subsets [J]. Chin J Cell Stem Cell (Electronic Edition). 2017, vol. 7, pp. 168-172.

[2] Yamaguchi T, Wing JB, Sakaguchi S. et al. Two modes of immune suppression by Foxp $3(+)$ regulatory $\mathrm{T}$ cellsunder inflammatory or non-inflammatory conditions [J]. Semin Immunol. 2011, vol. 23, pp. 424-430.

[3] Durand M, Lacoste P, Danger R, et al. High circulating CD4+CD25hiFOXP3+T-cell subpopulation early after lungtrans-plantation is associated with development of bronchiolitis obliterans syndrome [J]. J Heart Lung Transplant. 2018, vol. 37, pp. 770-781.

[4] Shi GX, Zeng Q, Li W, et al. Progress of CD4+CD25+Foxp3+regulatory $\mathrm{T}$ cell in autoimmune diseases [J]. J Immunological. 2016, vol. 32, pp. 1092-1095.

[5] Sakaguchi S, Sakaguchi N, Asano M, et al. Immunologic self-tolerance maintained by activated $\mathrm{T}$ cells expressing IL-2 receptor alpha-chains (CD25). Breakdown of a single mechanism of self-tolerance causes various autoimmune diseases [J]. J Immunol, 1995, vol. 115, pp. 1151-1164.

[6] Sakaguchi S, Sakaguchi N, Masanao A, et al. Immunologic selftolerance maintained by activated $\mathrm{T}$ cells expressing IL-2receptor alphachains (CD25) [J]. J Immunol, 2011, vol. 186, pp. 3808-3821.

[7] Henríquez C, Morán G, Carrasco C, et al. Modulatory role of regulatory $\mathrm{T}$ cells in a murine model of severe equine asthma [J]. BMC Vet Res, 2017, vol. 13, pp. 117.

[8] He X, Landman S, Bauland SC, et al. A TNFR2-agonist facilitates high purity expansion of human low purity treg cells [J]. PLoS One, 2016, vol. 11, pp. e0156311.

[9] Wang H, Li L, Zhang Y, et al. Expression and significance of CD4(+) CD25(+) CD127 (-) regulatory T cells in peripheral blood of patients with different phenotypes of Guillain-Barré syndrome $[\mathrm{J}]$. Int $\mathrm{J}$ Clin Exp Med, 2015, vol. 8, pp. 19126-19131.

[10] Glimire B, Gulina K. Research Progress of regulatory t cells and immunity of gynecologic malignant tumor [J]. Oncology Progress, 2011, vol. 9, pp. 172-176.

[11] Li Z, Ping Y, Yu Z, et al. Dynamic changes in CD45RA(-) Foxp3(high) regulatory T-cells in chronic hepatitis $\mathrm{C}$ patients during antiviral therapy [J]. Int J Infect Dis. 2016, vol. 45, pp. $5-12$.

[12] Son BR, Kim JY. Association of CD4(+) CD25(+) FoxP3(+) regulatory $\mathrm{T}$ cells with natural course of childhood chronic immune thrombocytopenic purpura [J]. Korean J Pediatr, 2015, vol. 58 , pp. $178-182$.
[13] Wang J, Yang J. Identification of CD4+CD25+CD127regulatory $\mathrm{T}$ cells and CD14+HLA-DR-/low myeloid-derived suppressor cells and their roles in the prognosis of breast cancer [J]. Biomed Rep, 2016, vol. 5, pp. 208-212.

[14] Mohammadnia-Afrouzi M, Zavaran Hosseini A, Khalili A, et al. Decrease of CD4(+) CD25(+) CD127(low) FoxP3(+) regulatory $\mathrm{T}$ cells with impaired suppressive function in untreated ulcerative colitis patients [J]. Autoimmunity. 2015, vol. 48 , pp. 556-561.

[15] Jin JO, Han X, Yu Q. Interleukin-6 induces the generation of IL-10-producing Tr1 cells and suppresses autoimmune tissue inflammation [J]. J Autoimmun. 2013, vol. 40, pp. 28-44.

[16] Quatromoni JG, Suzuki E, Okusanya O, et al. The timing of TGF- $\beta$ inhibition affects the generation of antigen-specific CD8+ T cells [J]. BMC Immunol. 2013, vol. 14, pp. 30.

[17] Cowan JE, Jenkinson WE, Anderson G. Thymus medulla fosters generation of natural Treg cells, invariant $\gamma \delta \mathrm{T}$ cells, and invariant NKT cells: what we learn from intrathymic migration [J]. Eur J Immunol. 2015, vol. 45, pp. 652-660.

[18] Qiao YC, Pan YH, Ling W, et al. The Yin and Yang of regulatory $\mathrm{T}$ cell and therapy progress in autoimmune disease [J]. Autoimmun Rev. 2017, vol. 16, pp. 1058-1070.

[19] Ye L, Goodall JC, Zhang L, et al. TCR usage, gene expression and function of two distinct FOXP3(+) Treg subsets within CD4(+) CD25(hi) T cells identified by expression of CD39 and CD45RO [J]. Immunol Cell Biol. 2016, vol. 94, pp. 293-305.

[20] $\mathrm{Lu} \mathrm{J}$, Meng H, Zhang A, et al. Phenotype and function of tissueresident unconventional Foxp3expressing CD4+ regulatory T cells [J]. Cell Immunol, 2015, vol. 297, pp. 53-59.

[21] Talaat RM, Elmaghraby AM, Barakat SS, et al. Alterations in immune cell subsets and their cytokine secretion profile in childhood idiopathic thrombocytopenic purpura (ITP) [J]. Clin Exp Immunol, 2014, vol. 176, pp. 291-300.

[22] Gulimire BRJ, Gulina KEB, Research Progress of regulatory t cells and immunity of gynecologic malignant tumor [J]. Oncology Progeress, 2011, vol. 9, pp. 172-176.

[23] Chraa D, Naim A, Olive D, et al. T lymphocyte subsets in cancer immunity: Friends or foes [J]. J Leukoc Biol. 2019, vol. 105, pp. 243-255.

[24] Scurr M, Ladell K, Besneux M, et al. Highly prevalent colorectal cancer-infiltrating LAP $^{+}$Foxp $3^{-\mathrm{T}}$ cells exhibitmore potent immunosuppressive activity than Foxp3+regulatory $\mathrm{T}$ cells [J]. Mucosal Immunol, 2014, vol. 7, pp. 428-439.

[25] Gandhi R, Farez MF, Wang Y, et al. Cutting edge: human latency-associated peptide $+\mathrm{T}$ cells: a novel regulatory $\mathrm{T}$ cell subset [J]. J Immunol. 2010, vol. 184, pp. 4620-4624.

[26] Noyan F, Lee YS, Zimmermann K, et al. Isolation of human antigen-specific regulatory $\mathrm{T}$ cells with high suppressive function [J]. Eur J Immunol, 2014, vol. 44, pp. 2592-2602.

[27] Edwards JP, Fujii H, Zhou AX, et al. Regulation of the expression of GARP/latent TGF- $\beta 1$ complexes on mouse T cells and their role in regulatory $\mathrm{T}$ cell and Th17differentiation [J]. Immunol J, 2013, vol. 190, pp. 5506-5515.

[28] Dancer R, Sansom DM. Regulatory $\mathrm{T}$ cells and COPD [J]. Thorax, 2013, vol. 68, pp. 1176-1178. 
[29] Kabir S. The role of interleukin-17 in the Helicobacter pylori induced infection and immunity [J]. Helicobacter. 2011, vol. 16 , pp. $1-8$.

[30] Galgani M, Insabato L, Calì G1, et al. Regulatory T cells, inflammation, and endoplasmic reticulum stress in women with defective endometrial receptivity [J]. Fertil Steril. 2015, vol. 103, pp. 1579-1586. e1.

[31] Liu JQ, Liu YH, Chen X, et al. Research progress of $\mathrm{CD} 4{ }^{+} \mathrm{CD} 25^{+} \mathrm{FoxP}^{+}{ }^{+}$regulatory $\mathrm{T}$ cells in tumorimmunosuppressive [J]. J Practical Oncology. 2016 vol. 30 , pp. 555-559.

[32] Zhao QH, Liang R, Li D, et al. Advances in researches on Foxp3+ regulatory $\mathrm{T}$ cell differentiation and its functional stability [J]. Acta University Atis Med Nanjing (Natural Science) 2017, vol. 37, pp. 1-9.

[33] Cheng AR, Cheng Y, Sun BL. Regulatory T Cells and Its Mechanism of Immunosuppression [J]. Chin J Clin Neurosci, 2014 vol. 22, pp. $438-444$

[34] Lu Y, Wang X, Gu J, et al. iTreg induced from CD39 (+) naive $\mathrm{T}$ cells demonstrate enhanced proliferate and suppressive ability [J]. Int Immunopharmacol. 2015, vol. 286, pp. 925-930.

[35] Hori S. Lineage stability and phenotypic plasticity of Foxp $3^{+}$regulatory T cells [J]. Immunol Rev, 2014, vol. 259, pp. 159-172.

[36] Kalia V, Penny LA, Yuzefpolskiy Y, et al. Quiescence of Memory CD8 (+) T Cells Is Mediated by Regulatory T Cells through Inhibitory Receptor CTLA-4 [J]. Immunity, 2015, vol. 42, pp. 1116-1129.

[37] Botta D, Fuller MJ, Marquez-Lago TT, et al. Dynamic regulation of $\mathrm{T}$ follicular regulatory cell responses by interleukin 2 during influenza infection [J]. Nat Immunol, 2017, vol. 18, pp. 1249-1260.

[38] Wei W, Luo M, Mao Q, et al. Research progress on the relationship between regulatory $\mathrm{T}$ cells and lung cancer $[\mathrm{J}]$. $\mathrm{J}$ Med Guizhou. 2018, vol. 42, pp. 284-287.

[39] Linterman MA, Denton AE. Treg cells and CTLA-4: the ball and chain of the germinal center response [J]. Immunity, 2014, vol. 41 , pp. 876-878.

[40] Ye Q, Zhang M, Wang Y, et al. Sirtinol regulates the balance of Th17/Treg to Prevent allog raft rejection [J]. Cell Biosci. 2017, vol. 7, pp. 55.

[41] Peterson RA. Regulatory T-cells: diverse phenotypes integral to immune homeostasis and suppression [J]. Toxicol Pathol, 2012, vol. 40, pp. 186-204.

[42] Tsai YG, Niu DM, Yang KD, et al. Functional defects of CD46-induced regulatory $\mathrm{T}$ cells to suppress airway inflammation in mite allergic asthma [J]. Lab Invest, 2012, vol. 92, pp. 1260-1269.

[43] Josefowicz SZ, Lu LF, Rudensky AY. Regulatory T cells: mechanisms of differentiation and function [J]. Annu Rev Immunol, 2012; vol. 30, pp. 531-564.

[44] Olson BM, Jankowska-Gan E, Becker JT, et al. Human prostate tumor antigen-specific $\mathrm{CD} 8+$ regulatory $\mathrm{T}$ cells are inhibited by CTLA-4 or IL-35 blockade [J]. J Immunol, 2012, vol. 189 , pp. 5590-5601.

[45] Yeh CH, Nojima T, Kuraoka M, et al. Germinal center entry not selection of $\mathrm{B}$ cells is controlled by peptide-MHCII complex density [J]. Nat Commun, 2018, vol. 9, pp. 1-11.

[46] Anderson AC, Joller N, Kuchroo VK. Lag-3, Tim-3, and TIGIT: Co-inhibitory Receptors with Specialized Functions in Immune Regulation [J]. Immunity. 2016, vol. 44, pp. 989-1004.

[47] Jiang T, Sun Y, Yin Z, et al. Research progress of indoleamine 2, 3-dioxygenase inhibitors [J]. Future Med Chem. 2015, vol. 7, pp. $185-201$.

[48] Christianson HC, Svensson KJ, van Kuppevelt TH, et al. Cancer cell exosomes depend on cell-surface heparan sulfate proteoglycans for their internalization and functional activity [J]. Proc Natl Acad Sci USA, 2013, vol. 110, pp. 17380-17385.

[49] Cao QJ, Zhou Yi C, Wang JY. Immunoregulation and progress of Treg cell-derived exosomes in disease research [J]. Chin J Immunol, 2018, vol. 34, pp. 1268-1273.

[50] Agarwal A, Fanelli G, Letizia M, et al. Regulatory $\mathrm{T}$ cell-derived exosomes: possible therapeutic and diagnostictools in transplantation [J]. Front Immunol, 2014, vol. 5, pp. 555 .

[51] Chatila TA, Williams CB. Regulatory T cells: exosomes deliver tolerance [J]. Immunity, 2014, vol. 41, pp. 3-5.

[52] Aiello S, Rocchetta F, Longaretti L, et al. Extracellular vesicles derived from $\mathrm{T}$ regulatory cells suppress $\mathrm{T}$ cell proliferation and prolong allograft survival [J]. Sci Rep, 2017, vol. 7, pp. 11518 .

[53] Biaggio RT, Abreu-Neto MS, Covas DT, et al. Serum-free suspension culturing of human cells: adaptation, growth, and cryopreservation [J]. Bioprocess Biosyst Eng, 2015, vol. 38, pp. 1495-507.

[54] Muller L, Mitsuhashi M, Simms P, et al. Tumor-derived exosomes regulate expression of immune function-related genes in human T cell subsets [J]. Sci Rep, 2016, vol. 6, pp. 20254.

[55] Okoye IS, Coomes SM, Pelly VS, et al. MicroRNA-containing T-regulatory-cell-derived exosomes suppress pathogenic T helper 1 cells [J]. Immunity, 2014. vol. 41, pp. 89-103.

[56] Zhang SP, Ni XH, Lu L. Progress on exosome and effect on regulatory $\mathrm{T}$ cells $[\mathrm{J}]$. Chin $\mathrm{J}$ Immunol, 2018, vol. 34, pp. $450-453,459$.

[57] Siebel C, Lendahl U. Notch Signaling in Development, Tissue Homeostasis and Disease [J]. Physiol Rev. 2017, vol. 97, pp. 1235-1294.

[58] Tegegn TZ, De Paoli SH, Orecna M, et al. Characterization of procoagulant extracellular vesicles and platelet membrane disintegration in DMSO-cryopreserved platelets [J. J Extracell Vesicles, 2016, vol. 5, pp. 30422.

[59] Charbonnier LM, Wang S, Georgiev P, et al. Control of peripheral tolerance by regulatory $\mathrm{T}$ cell-intrinsic Notch signaling [J]. Nat Immunol, 2015, vol. 16, pp. 1162-1173.

[60] Trehanpati N, Shrivastav S, Shivakumar B, et al. Analysis of Notch and TGF-â Signaling Expression in Different Stages of Disease Progression During Hepatitis B Virus Infection [J]. ClinTransl Gastroenterol, 2012, vol. 3, pp. e23. 
[61] Toker A, Engelbert D, Garg G, et al. Active demethylation of the Foxp3 locus leads to the generation of stable regulatory $\mathrm{T}$ cells within the thymus [J]. J Immunology, 2013, vol. 190, pp. 3180-3188. 